\title{
Investigation on a Novel Sensing Technology for the Detection of Blood Coagulation Based on Liquid Resonance Characteristics
}

\author{
Shengping Liu" a , Qi Zhou ${ }^{1}$ and Guoming Chen ${ }^{1}$ \\ ${ }^{1}$ Dept. of Biomedical Engineering, Chongqing University of Technology, China \\ a spliu@cqut.edu.cn
}

Keywords: Blood coagulation, Liquid resonance, Sensors, APTT.

\begin{abstract}
Based on the liquid resonance principle, a novel sensing technology for detecting the blood coagulation was proposed. The course of blood coagulation can alter its inherent properties, such as viscosity and density. As a result, liquid resonance frequency of blood will change with the blood agglomeration. A gold-plated Interdigital Electrode (IDE) was fabricated and a detection system was designed for the acquisition of the frequency. The activated partial thromboplastin time (APTT) was measured using the IDE and the detection system. For four volunteers, the activated partial thromboplastin time measured is $22-37$ seconds, which are consistent with the reference value 22-38 seconds. The results indicated that the liquid resonance frequency can characterize the course of blood coagulation.
\end{abstract}

\section{Introduction}

Due to the further understanding of the hemostasis and thrombosis and the application of electronic and computer technology in clinical detection, the detection technology of blood coagulation was improved rapidly. It can detect many significant parameters and index for the diagnosis, therapy and prognosis analysis of thrombosis and hemorrhagic diseases. Currently, the medical clinic usually adopted the optical turbidimetric method and the dual-magnetic circuit bead method to detect blood coagulation ${ }^{[1]}$. The detection results using the optical turbidimetric method are easily interfered by hemolysis, icterus, hyperlipoidemia, chylomicrons, and turbidity of liquid. And, for the dual-magnetic circuit bead method, test cups and beads need to treat particularly. Beads need to demagnetize and isolate from the magnetic field during the measurement. In addition, the arc shape of the bottom of the test cup must match the magnetic circuit, which could directly affect the measuring sensitivity. A new detection method based on Quartz Crystal Microbalance (QCM) sensor was proposed and applied in recent. However, the QCM sensor is hard to estimate the initial point and the endpoint of blood coagulation. For the clinical application, it is also hard to eliminate the influence of ion concentration, protein content and electrical conductivity etc ${ }^{[2-4]}$. Thrombelastography (TEG) has been commonly used in clinical detection for blood diseases ${ }^{[5,6]}$. During testing, the relative movement between test probes and blood specimens could alter the local properties of blood clots surrounding the probe. Otherwise, the surface characteristics of the cup wall and the rotation control accuracy of the cup will affect the measuring results.

Based on our previous researches, liquid has a specific electric resonance frequency, which is associate with its inherent properties such as ingredient, $\mathrm{pH}$, conductivity, concentration, viscosity, density and dielectric constant, etc. Blood coagulation will alter the inherent properties of blood, such as viscosity and density. As a result, the electric resonance frequency of blood will change. So the alteration of electric resonance frequency of blood can characterize the course of blood coagulation. Therefore, a novel sensing technology based on the liquid electric resonance principle was proposed and applied to the blood coagulation detection. It would provide a fast, accurate and simple detection technology and method for the blood coagulation detection and disease diagnosis related thrombus. 


\section{Experimental Materials}

Thrombin activator was purchased from Shanghai Sun Biology Technology Co. Ltd. Other reagents include $\mathrm{CaCl}_{2}$, anticoagulant sodium citrate solution, distilled water and so on. All reagents were used without further purification. The plasma derived from venous blood.

A gold-plated Interdigital Electrode (IDE) was fabricated as Fig.1. The diameter of the IDE substrate is $10 \mathrm{~mm}$. A detection system was designed and built. The configuration diagram of the detection system was showed in Fig. 2. Oscillating circuits generate a high-frequency square wave to apply to the IDE. Regulation circuits collect the resonance signal. MCU acquires the frequent value and transmit it to the computer (PC) via RS232 port. Computer performs the data processing, analysis, storage and display, etc.

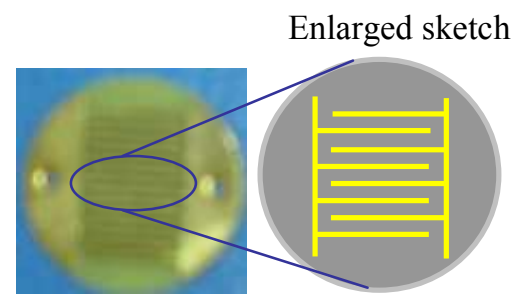

Fig. 1. IDE electrode

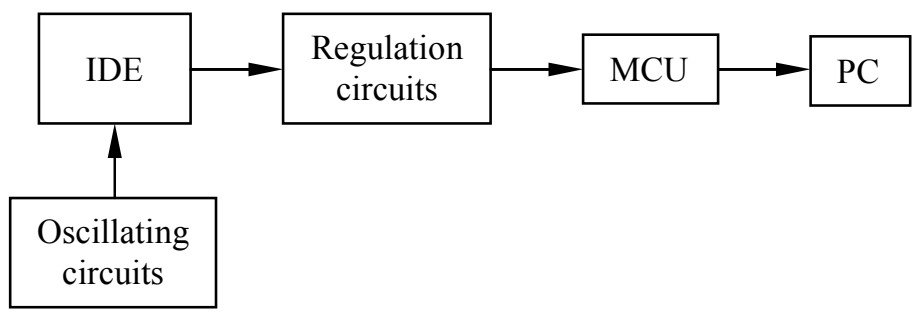

Fig. 2. The configuration diagram of the detection system

\section{Experiments}

The relationships between the liquid resonance frequency and the concentration of non-polar and polar solutions were investigated, respectively. The alcohol and $\mathrm{KCl}$ solution with different concentration were used for the investigation. The test results are shown in Fig.3. Within the specific limits of concentration, the liquid resonance frequency can characterize the concentration of solution from Fig.3. Viscosity and density of solutions are connected with the concentration, which will cause changes to of the liquid resonance frequency.
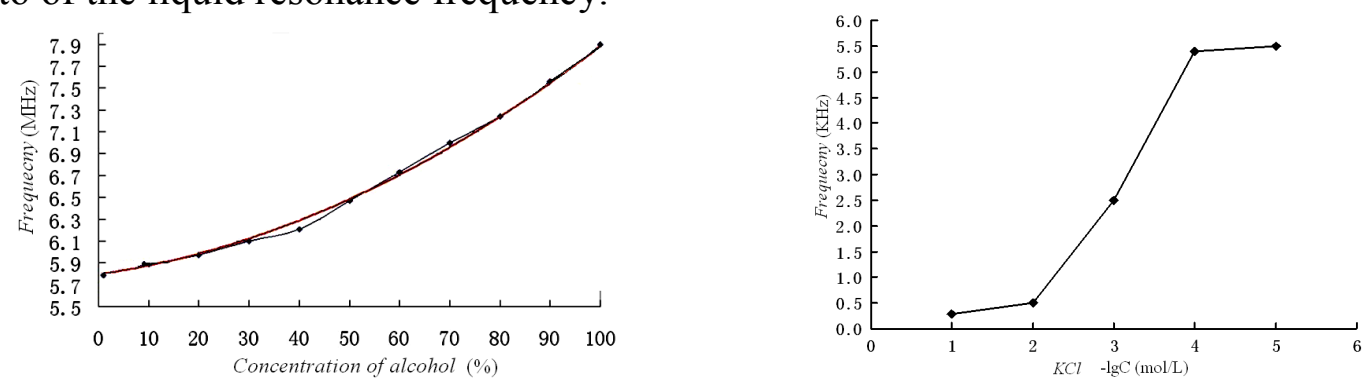

Fig. 3. The corresponding between the liquid resonance frequency and concentration

Four normal volunteers participated in the test of activated partial thromboplastin time (APTT), which is a key parameter of the acquired deficiency of coagulation factor VIII, IX, XI and the heparin. Volunteers' physical condition was listed in Table 1.

Blood specimens were collected from volunteers' vein. $90 \mu \mathrm{l}$ vein blood of volunteers was put into a plastic test tube which was installed $0.109 \mathrm{~mol} / \mathrm{L}$ anticoagulant sodium citrate solution $10 \mu \mathrm{l}$, respectively. After enough immingle, centrifugalize 15 minutes, $3000 \mathrm{rpm}$ at $8^{\circ} \mathrm{C}$. The upper yellow fluid, namely plasma, was collected and kept at $2-8^{\circ} \mathrm{C}$. It was noted that it must complete the plasma sample test within 6 hours.

Table 1 Physical condition of four volunteers

\begin{tabular}{c|c|l}
\hline Volunteer No. & Sex & \multicolumn{1}{|c}{ Physical condition of volunteers } \\
\hline 1 & Male & Healthy, $180 \mathrm{~cm}$ in height, $75 \mathrm{~kg}$ in weight \\
2 & Male & Healthy, $170 \mathrm{~cm}$ in height, $65 \mathrm{~kg}$ in weight \\
3 & Male & Healthy, $173 \mathrm{~cm}$ in height, $67 \mathrm{~kg}$ in weight \\
4 & Female & Healthy, $165 \mathrm{~cm}$ in height, $48 \mathrm{~kg}$ in weight \\
\hline
\end{tabular}


For APTT analysis, the IDE was fitted in the detection system firstly. $5 \mu 1$ plasma sample and $5 \mu 1$ thrombin activator solution were dropped on the IDE for incubating $3 \mathrm{~min}$ at $37^{\circ} \mathrm{C}$. The detection system real-timely monitors the resonance frequency of the IDE. When the resonance frequency trends to be stable, $5 \mu 10.025 \mathrm{~mol} / \mathrm{L} \mathrm{CaCl}_{2}$ solution was added to initiate a blood clotting response. The resonance frequency of the IDE was collected continuously at least 100s until the frequency become stable. All collected signals were transmitted to the computer for further analyzing and recording.

The IDE can reuse after cleared blood clots. Before reusing, it must be rinsed at least 3 times using distilled water, and then immersed in anhydrous alcohol for 30min; finally, the IDE was blown to dry with $\mathrm{N}_{2}$.

\section{Results and Discussions}

For plasma with the thrombin activator, $\mathrm{Ca}^{2+}$ will contribute the fibrinogen transformed into the insoluble fibrin. As a result, the density and the viscosity of the reaction system continuously increase. Thus, the acquired resonance frequency using the IDE will constantly increase until the plasma clotted entirely. According to the response curve of resonance frequencies, the initial point and the endpoint of agglutination reaction can be determined. Further, APTT can be calculated based on the initial point and the endpoint of agglutination reaction. So, the response curve of liquid resonance frequency can reflect the course of agglutination reaction and coagulation of blood. The variation of resonance frequency during agglutination reaction and coagulation of blood of four volunteers was plotted in Fig.4.
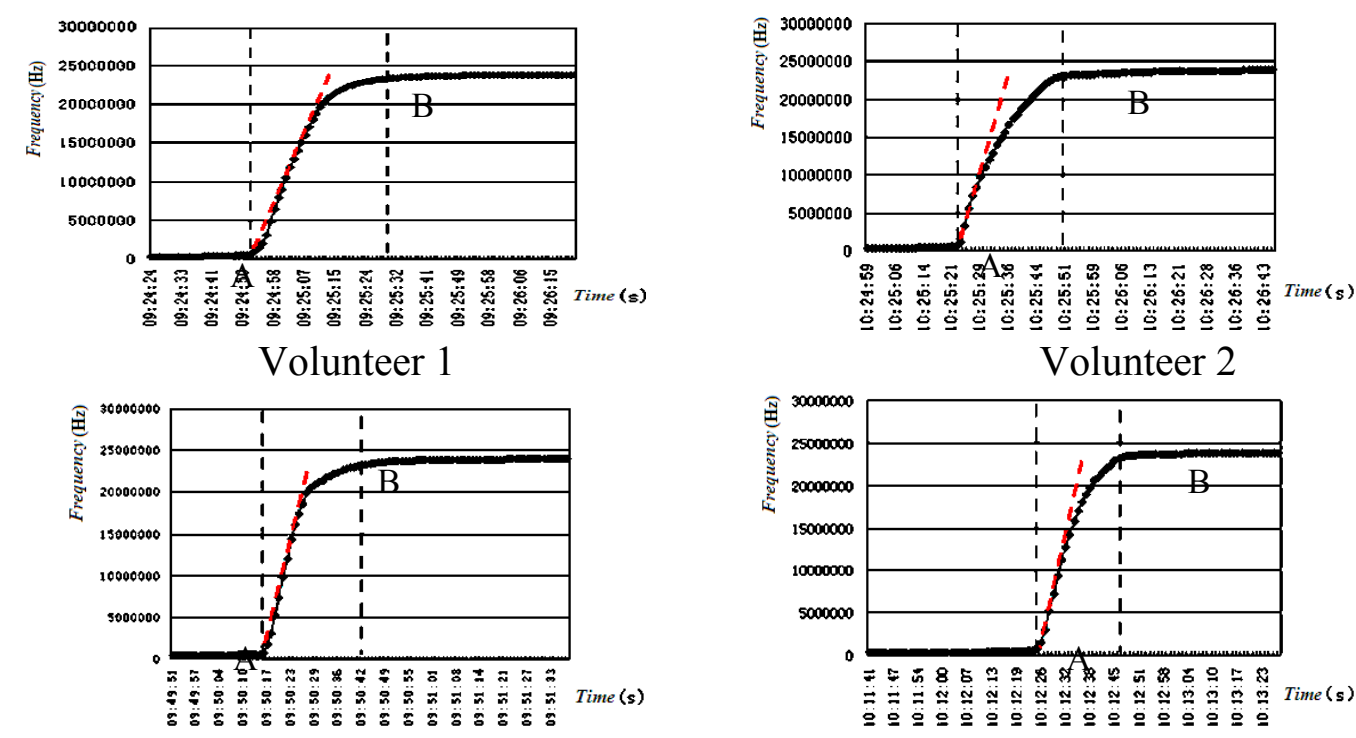

Volunteer 3

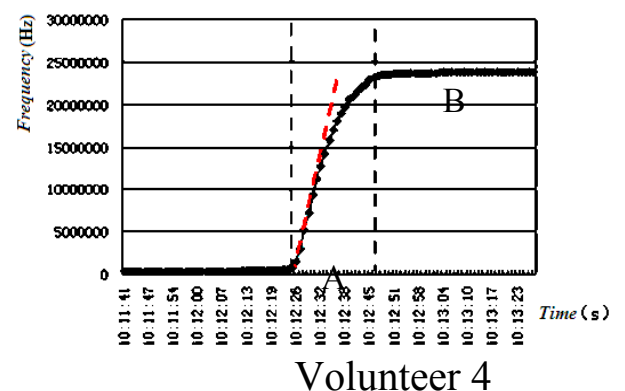

Fig. 4. The curve of the resonance frequency of APTT of four volunteers

From Fig. 4, after $\mathrm{CaCl}_{2}$ added, the curve of resonance frequency began to climb rapidly from $\mathrm{A}$ point, which is regarded as the initial point of agglutination reaction. A few seconds later, the curve trends to be steady from $\mathrm{B}$ point, which is regarded as the endpoint of agglutination reaction. So the period between A point and B point is the activated partial thromboplastin time. According to Fig.4, the APTT of four volunteers is determinate as Table 2. The reference value of APTT provided by Shanghai Sun Biology Technology Co. Ltd. is 22-38 seconds. Therefor, the proposed method in this paper can characterize the course of agglutination reaction and coagulation of blood. The slope of the tangent of the response curve through A point, as the red dashed line in Fig.4, can indicate the rapidity (kinetics) of fibrin build-up and cross-linking, that is, the speed of clot strengthening. The maximum amplitude of the curve represents the ultimate strength of the fibrin clot. 


\begin{tabular}{c|c} 
Table 2 & APTT of four volunteers \\
\hline Volunteer No. & APTT (s) \\
\hline 1 & 37 \\
2 & 29 \\
3 & 27 \\
4 & 22 \\
\hline
\end{tabular}

\section{Summary}

An Interdigital Electrode coated $\mathrm{Au}$ film and a detection system were uniquely designed and implemented for this research. The liquid electric resonance frequency can characterize the variation of the density and the viscosity during blood coagulating. A novel sensing technology for the detection of blood coagulation based on the liquid electric resonance property has been developed. APTT was analyzed used the novel sensing technology. The results indicated that the resonance frequency of liquid can reflect the course of blood coagulation. By this way, the analysis of blood coagulation becomes quicker and efficient. It will benefit to the diagnosis of blood diseases.

Since the sensing technology based on the liquid resonance principle is well-suited for the analysis of the inherent properties of liquid, the measurement of other key parameters of blood coagulation such as prothrombin time (PT) and thrombin time (TT) etc, is expected to be more excellent comparing to the conventional methods.

\section{Acknowledgment}

This work is supported by Natural Science Foundation of Chongqing (Granted No. CSTC 2009BB5051).

\section{References}

[1] YU Kui, LIN Guoqing, QU Zhe et al. The state of the art of the blood coagulation analyzers, Information of Medical Equipment. 12 (2001) 40-41

[2] ZHAO Meng, FU Wei-ling, CHEN Ming et al. Exponential decay regularity of frequency and endpoint determination by maximalcurvature in piezoelectric coagulation, Acta Academiae Medicinae Militaris Tertiae. 28 (2006) 311-314

[3] Cheng T J, Chang H C, Lin T M. A piezoelectric quartz crystal sensor for the determination of coagulation time in plasma and whole blood, Biosensors Bioelectronics. 13 (1998) 147-156

[4] Ganter M T, H ofer C K. Coagulation monitoring:current techniques and clinical use of viscoelastic point-of-care coagulation devices, Anesth Analg. 106(5) (2008) 1366-1375

[5] Dai Y, Lee A , Critchley L A, et a1. Does thromboelastography predict postoperative thromboem bolic events? A systematic review of the literature, Anesth Analg. 108(3) (2009) 734-742.

[6] Kalina U, StOhr H A, Bickhard H, et a1. Rotational thromboelastography for monitoring of fibrinogen concentrate therapy in fibrinogen deficiency. Blood Coagul Fibrinolysis. 19(8) (2008) 777-783. 